\title{
The radio spectrum and magnetic field structure of SNR HB3
}

\author{
W. B. Shi ${ }^{1,3}$, J. L. $\operatorname{Han}^{1}$, X. Y. Gao ${ }^{1}$, X. H. Sun ${ }^{1}$, L. Xiao ${ }^{1}$, P. Reich ${ }^{2}$, and W. Reich ${ }^{2}$ \\ ${ }^{1}$ National Astronomical Observatories, Chinese Academy of Sciences, Jia 20 DaTun Road, ChaoYang District, \\ Beijing 100012, PR China \\ e-mail: hjl@bao.ac.cn \\ 2 Max-Planck-Institut für Radioastronomie, Auf dem Hügel 69, 53121 Bonn, Germany \\ 3 School of Space Science and Physics, Shandong University at Weihai, 180 Cultural West Road, Shandong 264209, PR China
}

Received 29 April 2008 / Accepted 7 June 2008

\begin{abstract}
Context. Evidence for a spectral flattening of the supernova remnant (SNR) HB3 (G132.7+1.3) was recently claimed in the literature based on previously published total flux density data, and the flattening was further interpreted as the discovery of thermal bremsstrahlung emission in the shell of HB3.

Aims. A spectral flattening has never been observed from any SNR before. Reliable observations of HB3 at frequencies above $3000 \mathrm{MHz}$ are crucial to confirm such a spectral behaviour.

Methods. We extracted $4800 \mathrm{MHz}$ total intensity and polarisation data of HB3 from the Sino-German $6 \mathrm{~cm}$ polarisation survey of the Galactic plane made with the Urumqi $25 \mathrm{~m}$ telescope, and analysed the spectrum of HB3, together with Effelsberg data at $1408 \mathrm{MHz}$ and $2675 \mathrm{MHz}$.

Results. We found an overall spectral index of HB3 of $\alpha=-0.61 \pm 0.06$ between $1408 \mathrm{MHz}$ and $4800 \mathrm{MHz}$, similar to the index at lower frequencies. There is no spectral flattening at high frequencies. We detected strong polarised emission from $\mathrm{HB} 3 \mathrm{at} 4800 \mathrm{MHz}$. Our $4800 \mathrm{MHz}$ data show a tangential field orientation in the HB3 shell.
\end{abstract}

Key words. ISM: supernova remnants - ISM: individual objects: HB3 - radio continuum: ISM

\section{Introduction}

Radio emission from a supernova remnant (SNR) originates from synchrotron emission of relativistic electrons gyrating in the enhanced magnetic fields in its shell or filaments. The observed radio spectrum of a SNR generally follows a power-law $\left(S_{v} \propto v^{\alpha}\right.$, where $\alpha$ is the spectral index). A definite case of a spectral break was detected for the SNR S147 (Fürst \& Reich 1986; Xiao et al. 2008).

HB3 is a large $\left(1.5 \times 2^{\circ}\right)$ and evolved SNR, with an age of about $30000 \mathrm{yr}$ (Lazendic \& Slane 2006). It is located at the edge of the HII regions W3/W4 and belongs to a complex of molecular clouds, star-formation and HII regions (W3/W4/W5) (e.g., Digel et al. 1996) in the Perseus arm at a distance of about $2.2 \mathrm{kpc}$ (Routledge et al. 1991; Xu et al. 2006). Although the SNR HB3 partly overlaps with the adjacent HII regions, W3/W4, in the low-resolution radio maps, signs of a real interaction, such as a deformed shell in the high-resolution maps or $\mathrm{OH}$ masers in the joint region from the HB3 shock, have not been detected (Koralesky et al. 1998; Kothes et al. 2006). Nevertheless, enhanced radio emission has been observed at all radio bands in the southeastern regions of HB3 (towards the lower-left in Fig. 1).

Previous estimates of the spectral index of HB3 were made from flux densities determined at frequencies up to $3 \mathrm{GHz}$, mostly without point source subtraction. The spectral index of HB3 was quoted to have values of $\alpha=-0.64 \pm 0.01$ (Fesen et al. 1995), $\alpha=-0.56 \pm 0.03$ (Green 2007), $\alpha=-0.60 \pm$ 0.04 (Landecker et al. 1987), and $\alpha=-0.66 \pm 0.02$ (Kothes et al. 2006). Flux-density estimates of HB3 from low-resolution observations are uncertain, especially at high frequencies, because of the partial overlap with W3/W4 (see Green 2007).

Recently, Tian \& Leahy (2005) obtained images of HB3 at $408 \mathrm{MHz}$ and $1420 \mathrm{MHz}$ from the Canadian Galactic Plane Survey (CGPS: Taylor et al. 2003) with compact sources and diffuse background emission subtracted. They derived a spectral index for HB3 of $\alpha=-0.34 \pm 0.25$ between these two frequencies (Tian \& Leahy 2005, 2006, erratum). This result was confirmed by the TT-plot method. They also found a surprising result: a spectral flattening of the overall HB3 spectrum above $1 \mathrm{GHz}$, derived from all published flux density data from $38 \mathrm{MHz}$ to $3900 \mathrm{MHz}$. Urošević et al. (2007) fitted the spectrum using a two-component model of synchrotron emission and thermal bremsstrahlung emission. They concluded that the flattening is evidence for the detection of thermal bremsstrahlung emission produced in the shell of the SNR. However, there are severe problems in the determination of flux density data of HB3, as discussed by Green (2007). The uncertainties in the quoted flux density data, especially at high frequencies $(1 \mathrm{GHz}$ to $3 \mathrm{GHz})$, are large, therefore the spectral flattening has been discredited by Green (2007). Radio observations at even higher frequencies are required to clarify this extraordinary spectral behaviour.

The ongoing Sino-German $\lambda 6 \mathrm{~cm}$ polarisation survey of the Galactic plane (Sun et al. 2007) has already covered the HB3 area. In this paper, we analyse our $\lambda 6 \mathrm{~cm}(4800 \mathrm{MHz})$ data together with $1408 \mathrm{MHz}$ and $2675 \mathrm{MHz}$ data from the Effelsberg Galactic plane surveys (Reich et al. 1997; Fürst et al. 1990a) to determine the high-frequency spectrum of HB3. Furthermore, instead of using radio emission of the entire SNR, where its eastern part obviously suffers from contamination or confusion by 

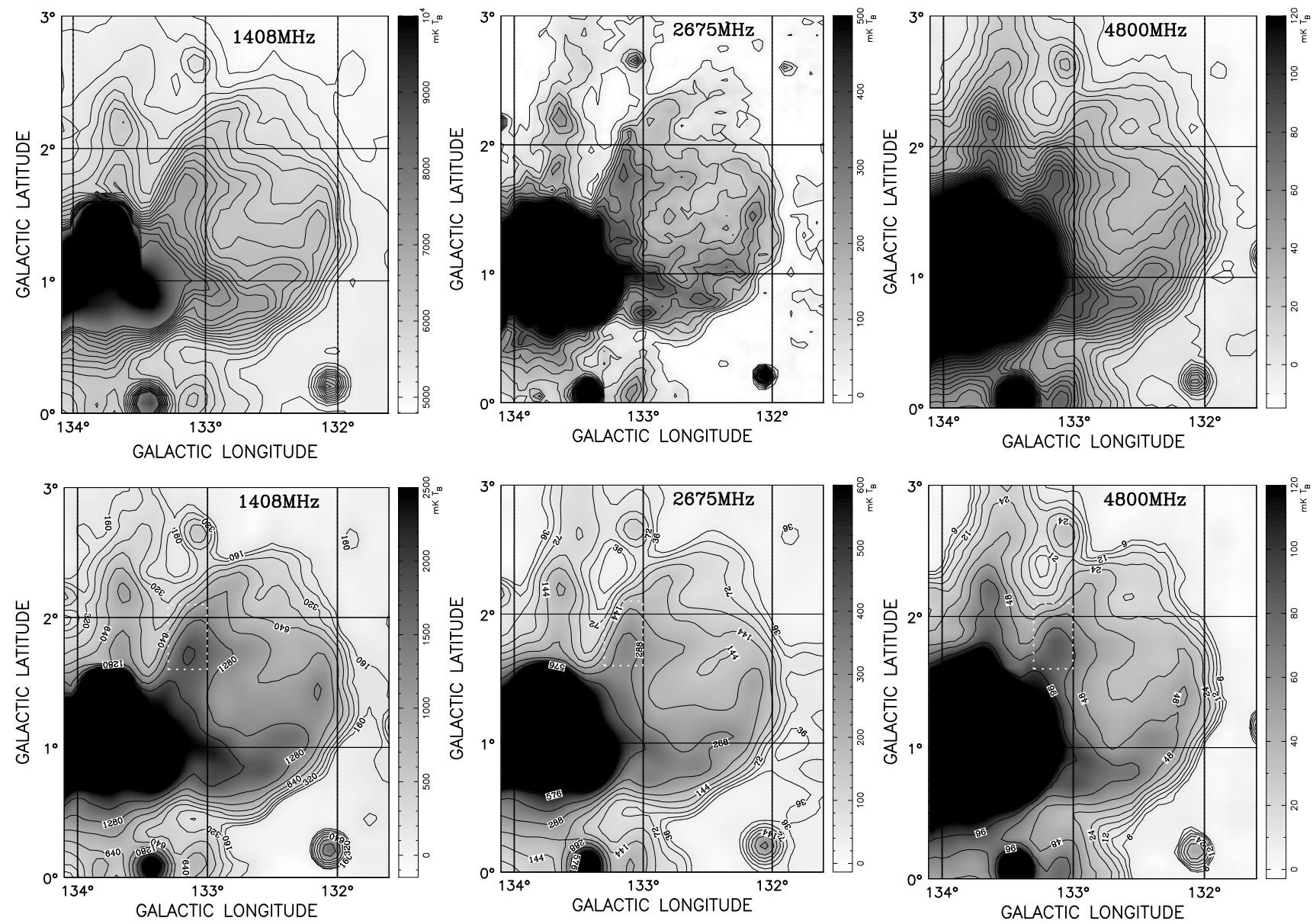

Fig. 1. HB3 maps at $1408 \mathrm{MHz}, 2675 \mathrm{MHz}$, and $4800 \mathrm{MHz}$. The top row shows the data after the subtraction of point-like sources in the area of HB3 and 10' outside. The angular resolution at $1408 \mathrm{MHz}, 2675 \mathrm{MHz}$, and $4800 \mathrm{MHz}$ is 9'.4, 4.3 , and 9'.5, respectively. The bottom row displays the same data convolved to a common angular resolution of $10^{\prime}$.

thermal emission, we limit our study to the part being almost free of distortions. The first detection of polarised emission of HB3 at $\lambda 6 \mathrm{~cm}$ will also be briefly discussed.

\section{Radio data of the HB3 region}

The $1408 \mathrm{MHz}$ data are extracted from the Galactic plane surveys ${ }^{1}$ observed with the Effelsberg $100 \mathrm{~m}$ telescope (Reich et al. 1997). The $2675 \mathrm{MHz}$ data were observed in 1998 in the same way as early 11-cm survey (Fürst et al. 1990a) but with a better receiver and higher sensitivity. The angular resolutions are 9'.4 and 4.3, respectively. The new $4800 \mathrm{MHz}$ data were observed with the Urumqi $25 \mathrm{~m}$ radio telescope with an angular resolution of 9'.5. Observations were made by scans over $10^{\circ}$ in length in both Galactic longitude and latitude, which is the limiting size for a reliable flux density determination of an object. Technical details of the $4800 \mathrm{MHz}$ observations and data processing are described by Sun et al. (2007).

For a more accurate determination of the radio emission of HB3 at these three frequencies, we first identified and then subtracted unresolved point sources above the local surrounding level of diffuse emission in the region of HB3 and 10' outside. In total, 107 point sources have been identified on the highresolution CGPS maps at $408 \mathrm{MHz}$ and $1420 \mathrm{MHz}$ (Taylor et al. 2003), and their spectral indices were determined if visible at

${ }^{1}$ http://www.mpifr.de/survey.html both frequencies. Their fluxes at $2675 \mathrm{MHz}$ and $4800 \mathrm{MHz}$ were obtained by extrapolation. However, the fluxes of seven compact sources at $2675 \mathrm{MHz}$ have been taken from Fürst et al. (1990b) and of eight point-like sources at $4800 \mathrm{MHz}$ from the 87GB catalogue of Gregory \& Condon (1991). The resulting maps are shown in the upper part of Fig. 1.

The large-scale Galactic background/foreground emission is obviously not uniform in the maps. Clearly it is stronger in the eastern part than in the western part, and stronger in the south than in the north. We subtracted a "twisted" base-level, so that the intensity level of the area surrounding HB3 is close to zero, hence, removed the large-scale Galactic emission. We then convolved all maps to the same $10^{\prime}$ angular resolution for the spectral index analysis.

\section{Spectral analysis of HB3}

The boundary between HB3 and the adjacent HII regions can be fairly well defined in high-resolution low-frequency maps (Green 2007), but this is difficult for our low-resolution maps. To avoid the contamination problem that Green (2007) emphasised, the HB3 region west of $l=133^{\circ}$ and in an eastern box region (defined as $l=133^{\circ}$ to $133^{\circ} .3, b=1^{\circ} .6$ to $2^{\circ} .1$ ) are analysed quantitatively. No contamination from W3/W4 appears in these regions for all three frequencies (see Fig. 1).

Figure 2 shows that the integrated flux densities of the HB3 region west of $l=133^{\circ}$ decrease with frequency. The 


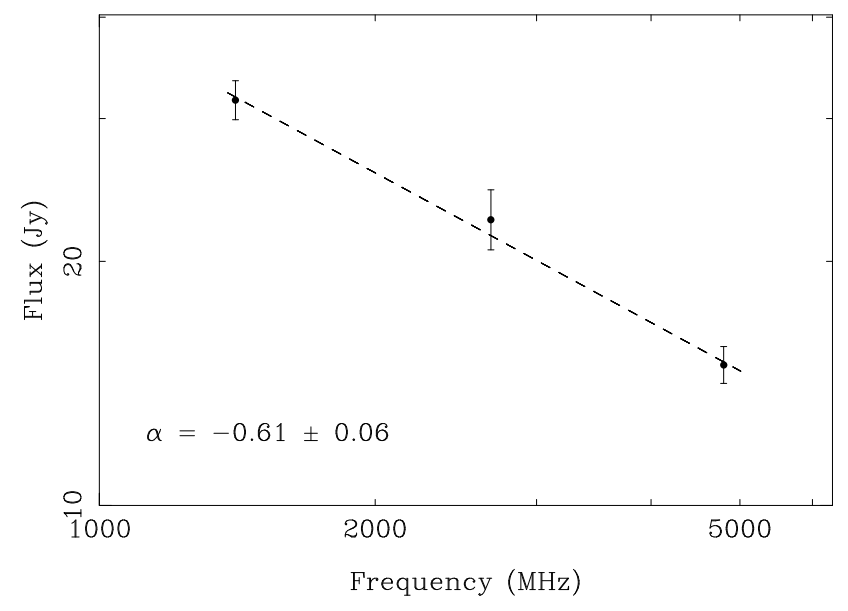

Fig. 2. Spectrum of the HB3 region west of $l=133^{\circ}$.

spectral index between $1408 \mathrm{MHz}$ and $4800 \mathrm{MHz}$ is $\alpha \sim-0.6$, consistent with the value Landecker et al. (1987) obtained from $408 \mathrm{MHz}$ and $1420 \mathrm{MHz}$ maps, and that from the low-frequency integrated flux densities in Kothes et al. (2006) and in Fig. 3 of Tian \& Leahy (2005).

The HB3 spectral behaviour can be verified by the so called TT-plot method (see Fig. 3), which largely reduces the influence of unrelated large-scale emission. Having convolved the maps to the same angular resolution, the pixel values at every $5^{\prime}$ sampling for a selected area are plotted against each other. The slope $\beta$ determined by linear regression is the brightness temperature spectral index $\left(T \propto v^{\beta}\right)$. The flux density spectral index, $\alpha$, is related to $\beta$ as $\beta=\alpha-2$. The $\alpha$ and $\beta$ values in Figs. 2 and 3 are consistent (see Table 1). The emission in the eastern box region has a spectral behaviour similar to the HB3 region west of $l=133^{\circ}$.

The basic assumption for a reliable TT-plot method is that the emission at each pixel of the extended object has the same spectral index between the two frequencies, though the intensity varies from pixel to pixel. This is the case for many SNRs, but is not always true. Deviations from the fitted slope of a TT-plot may indicate either a possible spectral deviation for a certain region or base-level fluctuations across the object. In Fig. 4, we show the resolved spectral index maps. The very central part of HB3 seems to have a steeper spectrum, although the low intensity there also implies a larger uncertainty in the spectral index.

\section{Polarised radio emission}

From our $4800 \mathrm{MHz}$ observations, we clearly detected polarised emission from HB3 for the first time (see Fig. 5). No polarised emission has been detected at lower frequencies, so Faraday rotation of the polarisation angles cannot be corrected. The polarised emission of HB3 at $4800 \mathrm{MHz}$ is strongest in the eastern shell with a percentage polarisation of up to $28 \%$. Some largescale, polarised diffuse Galactic foreground emission is also visible in the map.

Pulsar rotation measures in the direction of the Perseus arm are a few tens of $\mathrm{rad} \mathrm{m}^{-2}$ at maximum (Han et al. 1999). This results in a polarisation angle rotation of less than $20^{\circ}$ at $4800 \mathrm{MHz}$. A $90^{\circ}$ rotation of our observed $\boldsymbol{E}$ vectors roughly indicates the magnetic field orientation. Magnetic field lines seem to follow the shell, including the weak polarised emission in the western limb, which is a typical characteristic of an evolved SNR. A very exceptional area showing a radial
Table 1. Spectral indices of the HB3 regions.

\begin{tabular}{ccccc}
\hline \hline Freq. pairs & $\alpha_{133 w e s t}$ & $\beta_{133 \text { west }}^{\text {TT }}$ & $\alpha_{\text {east.box }}$ & $\beta_{\text {east.box }}^{\text {TT }}$ \\
\hline $1408 / 2675$ & $-0.51 \pm 0.16$ & $-2.64 \pm 0.09$ & $-0.49 \pm 0.25$ & $-2.69 \pm 0.02$ \\
$1408 / 4800$ & $-0.61 \pm 0.06$ & $-2.61 \pm 0.05$ & $-0.53 \pm 0.09$ & $-2.54 \pm 0.16$ \\
$2675 / 4800$ & $-0.71 \pm 0.18$ & $-2.57 \pm 0.20$ & $-0.58 \pm 0.28$ & $-2.32 \pm 0.10$ \\
\hline
\end{tabular}

magnetic field is located in the extreme southern part of the rim. It resembles a circular half shell with a diameter of $20^{\prime}$, centred at $(l, b)=(132.75,0.55)$ (see the right panel of Fig. 5, and also the $\mathrm{H} \alpha$ image, Fig. 8 of Fesen et al. 1995). Based on the distinct magnetic morphology, we suspect this may be another young SNR, rather than a limb of HB3. However, higherresolution polarisation observations are needed to clarify the nature of this feature.

In the central region, where complete depolarisation occurs in our $4800 \mathrm{MHz}$ map, ring-like X-ray emission has been detected by the Einstein satellite (for a comparison with the radio emission see Landecker et al. 1987; Venkatesan et al. 1984) and more recent ROSAT observations (see Fig. 11 in Lazendic \& Slane 2006).

\section{Discussion and conclusions}

We studied the spectrum of HB3 using maps at $1408 \mathrm{MHz}$, $2675 \mathrm{MHz}$, and $4800 \mathrm{MHz}$ for the regions of HB3 where no contamination from $\mathrm{W} 3 / \mathrm{W} 4$ is visible. We obtained a spectral index for HB3 between $1408 \mathrm{MHz}$ and $4800 \mathrm{MHz}$ of $\alpha=-0.61 \pm 0.06$, which is consistent with values obtained by Fesen et al. (1995), Landecker et al. (1987), Kothes et al. (2006). The TT-plot method was used to verify the spectrum. The new $4800 \mathrm{MHz}$ data directly show that the spectrum of HB3 is constant with $\alpha \sim-0.6$ from $1 \mathrm{GHz}$ to $5 \mathrm{GHz}$. There is no evidence for a spectral flattening to higher frequencies discussed earlier by Tian \& Leahy (2005). This conclusion is in entire agreement with Green (2007). Therefore, the thermal bremsstrahlung emission discussed by Urošević et al. (2007) does not exist in the HB3 areas not contaminated by thermal emission related to W3/W4.

Polarised radio emission is clearly detected, which indicates that the magnetic field is aligned with the shell of HB3, except for the southern spherical limb, which we suspect to be an independent feature.

Acknowledgements. The $\lambda 6 \mathrm{~cm}$ data were obtained with the receiver system from the MPIfR mounted at the Nanshan $25 \mathrm{~m}$ telescope at the Urumqi Observatory of NAOC. The authors are supported by the National Natural Science Foundation (NNSF) of China (10521001 and 10773016), the National Key Basic Research Science Foundation of China (2007CB815403), and the Partner group of the MPIfR at NAOC in the frame of the exchange program between MPG and CAS for many bilateral visits. We thank Dr. James Anderson for carefully reading the manuscript.

\section{References}

Digel, S. W., Lyder, D. A., Philbrick, A. J., Puche, D., \& Thaddeus, P. 1996, ApJ, 458,561

Fesen, R. A., Downes, R. A., Wallace, D., \& Normandeau, M. 1995, AJ, 110, 2876

Fürst, E., \& Reich, W. 1986, A\&A, 163, 185

Fürst, E., Reich, W., Reich, P., \& Reif, K. 1990a, A\&AS, 85, 691

Fürst, E., Reich, W., Reich, P., \& Reif, K. 1990b, A\&AS, 85, 805

Green, D. A. 2007, Bull. Astron. Soc. Ind., 35, 77

Gregory, P. C., \& Condon, J. J. 1991, ApJS, 75, 1011

Han, J. L., Manchester, R. N., \& Qiao, G. J. 1999, MNRAS, 306, 371

Koralesky, B., Frail, D. A., Goss, W. M., Claussen, M. J., \& Green, A. J. 1998, AJ, 116, 1323 

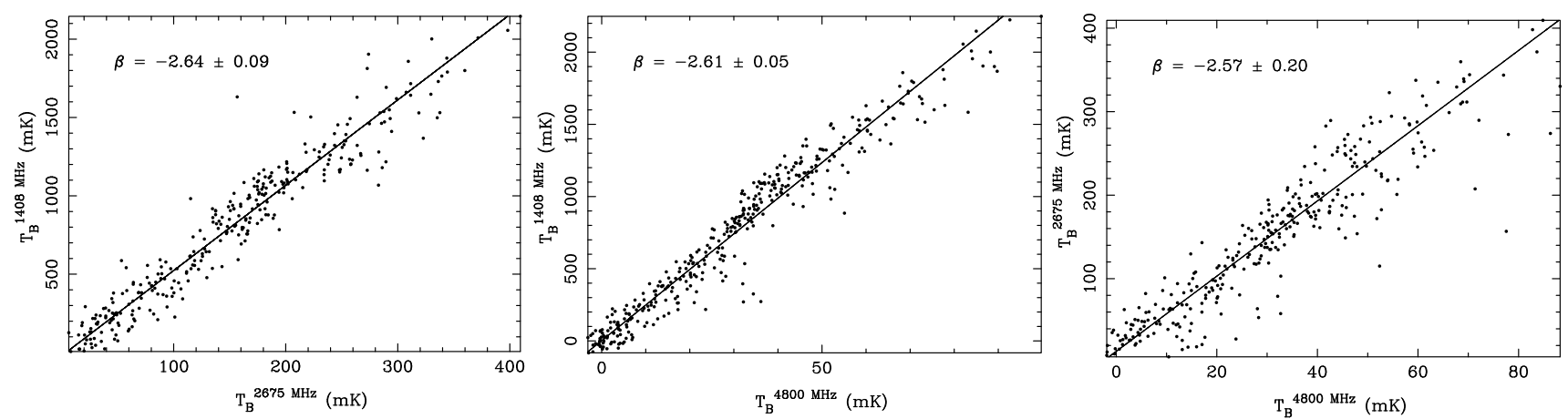

Fig. 3. TT-plots of the HB3 region west of $l=133^{\circ}$.
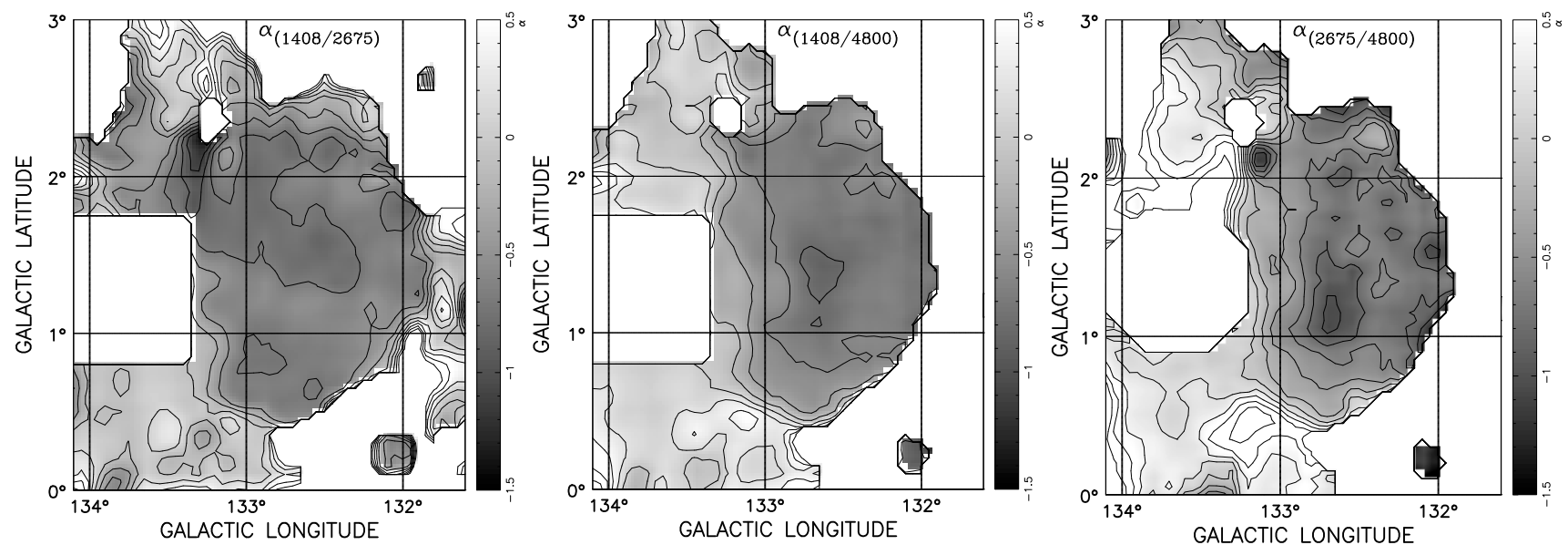

Fig. 4. Spectral index maps of HB3 calculated for three frequency pairs.
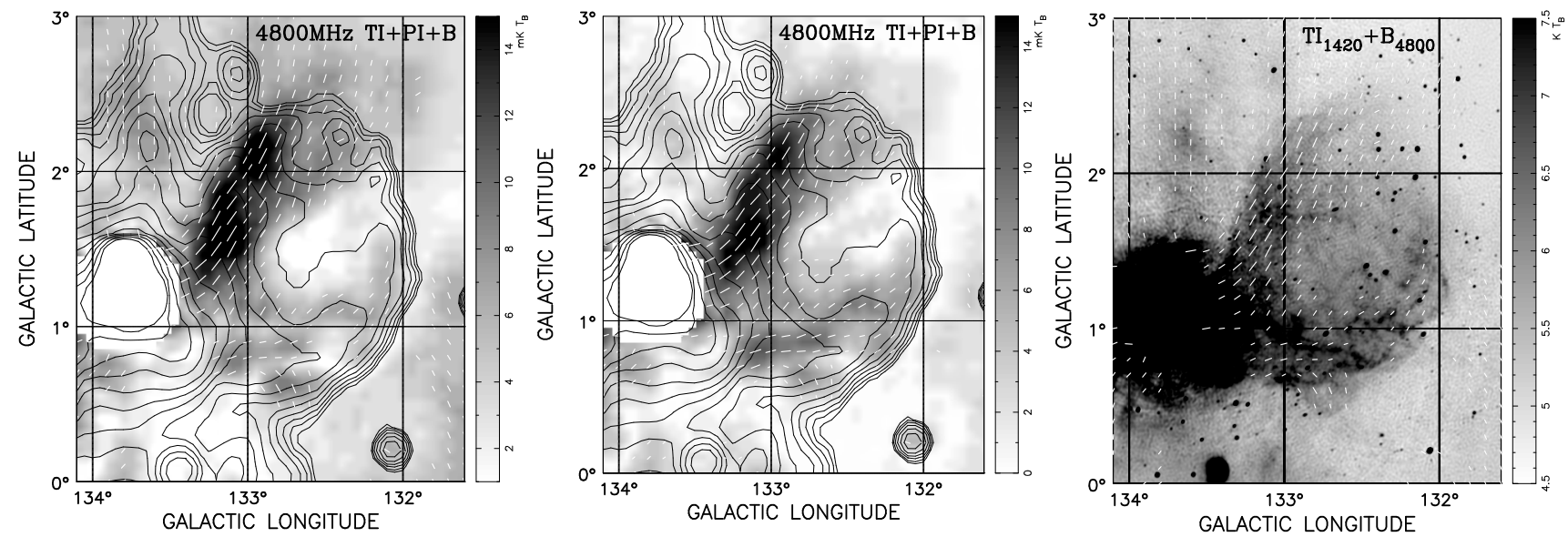

Fig. 5. Polarised emission from HB3 detected at $4800 \mathrm{MHz}$. Left panel: total intensity map at $4800 \mathrm{MHz}$ is displayed by contours, and the polarised intensity map is shown in grey-scale. The bars represent $\left(\boldsymbol{E}+90^{\circ}\right)$, roughly indicating the magnetic field orientation. The bar length is proportional to the polarised intensity. The diffuse Galactic emission is not subtracted. Middle panel: same as the left but diffuse Galactic polarised emission has been removed in the polarised intensity map. Right panel: polarisation angles $\left(\boldsymbol{E}+90^{\circ}\right) \simeq \boldsymbol{B}$ calculated from original maps at $4800 \mathrm{MHz}$ are plotted over the high-resolution $1420 \mathrm{MHz}$ total intensity map (grey-scale) from the Canadian Galactic Plane Survey (Taylor et al. 2003).

Kothes, R., Fedotov, K., Foster, T. J., \& Uyanıker, B. 2006, A\&A, 457, 1081 Landecker, T. L., Vaneldik, J. F., Dewdney, P. E., \& Routledge, D. 1987, AJ, 94, 111

Lazendic, J. S., \& Slane, P. O. 2006, ApJ, 647, 350

Reich, P., Reich, W., \& Fürst, E. 1997, A\&AS, 126, 413

Routledge, D., Dewdney, P. E., Landecker, T. L., \& Vaneldik, J. F. 1991, A\&A, 247,529

Sun, X. H., Han, J. L., Reich, W., et al. 2007, A\&A, 463, 993
Taylor, A. R., Gibson, S. J., Peracaula, M., et al. 2003, AJ, 125, 3145

Tian, W. W., \& Leahy, D. 2005, A\&A, 436, 187

Tian, W. W., \& Leahy, D. A. 2006, A\&A, 451, 991, erratum

Urošević, D., Pannuti, T. G., \& Leahy, D. 2007, ApJ, 655, L41

Venkatesan, D., Leahy, D. A., Galas, C. M. F., Naranan, S., \& Long, K. 1984, MNRAS, 208, 25P

Xiao, L., Fürst, E., Reich, W., \& Han, J. L. 2008, A\&A, 482, 783

Xu, Y., Reid, M. J., Zheng, X. W., \& Menten, K. M. 2006, Science, 311, 54 